\title{
What does 'holism' mean in Indigenous mental health?
}

\section{A review of the literature and suggestions for healthcare professionals}

\author{
Ranjana Bhattacharjee, Alana Maltby \\ Faculty Reviewer: Piotr Wilk (Department of Epidemiology \& Biostatistics), Martin Cooke (Department of Sociology \& Legal Stud- \\ ies, University of Waterloo)
}

\section{ABSTRACT}

In Canada, suicide and depression rates are much higher among Indigenous populations compared to the general population. Colonial practices, cultural discontinuity, marginalization, and oppression have led to many of the mental health issues faced by Indigenous populations today. Recent literature emphasizes the importance of culture in the treatment and prevention of mental health problems among Indigenous peoples. Unfortunately, Indigenous perspectives on mental health or wellness in the peer-reviewed literature are often limited. This review aims to incorporate the grey literature produced by Indigenous organizations to better describe what 'holism' is as it relates to the wellness of Indigenous peoples, and to identify some practical implications of this understanding for healthcare providers addressing the needs of Indigenous patients and clients.

\section{INTRODUCTION}

Suicide and depression rates in Canada are currently much higher among Indigenous populations compared to the general population. ${ }^{1}$ Mental health inequities have been linked to the many effects of colonization, including the relocation of communities, forced sedentarization, residential schools, and the ensuing breakup of Indigenous family structures, social isolation, and poverty. ${ }^{2-5}$

Recent literature has highlighted the importance of culture in the treatment and prevention of mental health problems among Indigenous peoples. ${ }^{5-7}$ However, the portrayal of Indigenous perspectives on mental health or healing practices in the peer-reviewed literature is often limited. Indigenous peoples' understandings of health are typically described as 'holistic' and often refer to a Medicine Wheel model, balancing the physical, mental, spiritual, and emotional aspects of life. However, a deeper understanding of Indigenous peoples' conceptualizations of mental health, what 'holism' might mean in the lives of patients, and suggestions for application in mental health practice, are needed.

Indigenous perspectives on mental health are more easily found in the grey literature produced by Indigenous organizations or research groups, using interviews and focus groups with Indigenous peoples, Elders, and service providers. The purpose of this paper is to incorporate this literature to better describe what is meant by 'holism' and to identify some practical implications of this understanding for healthcare professionals addressing the needs of Indigenous patients.

\section{HOLISM AS 'BALANCE'}

Notions of well-being are holistic, integrating the spiritual, emotional, physical, and mental dimensions of health. ${ }^{8-10}$ Balance between these four dimensions and within each of these spheres is necessary to maintain not only the health and wellness of a person, but also their family and community., 9, 10 This understanding of well-being is often placed in opposition to 'Western' perspectives that tend to dichotomize mental and physical health.

Spirituality and spiritual balance were given significance among the literature. Spiritual wellness among First Nations is achieved through connectedness to beliefs, values, and identity. ${ }^{2}$ The First Nations Mental Wellness Continuum (FNMWC) describes spiritual wellness as having 'hope for the future'. ${ }^{2}$ FNMWC is a Canada-wide framework addressing mental wellness among First Nations in Canada, built through collaboration of the Assembly of First Nations, Health Canada, and community mental health leaders. Among Métis women, well-being meant understanding the spiritual dimension, the importance of prayer, and traditional spiritual practices to gain inner strength and resilience. ${ }^{8}$ One study referred to the Inuit use of spirit possession as explanation for dramatic changes in individual behaviours. ${ }^{11}$

Emotional balance was found to be important in interviews with First Nations Elders, healthcare providers, and clients, some of whom believed that loss of emotional control reflected mental health problems while others thought that it was important to be comfortable with emotional outpouring. ${ }^{12}$ Métis women spoke of the importance of living daily life while managing emotions, including releasing negative emotions quickly, being emotionally selfaware, and accepting of the need to express emotions. ${ }^{8}$

In terms of the physical dimension of wellness, the FNMWC describes how the physical self is affected by a person's 'way of being', which includes daily activities such as employment and education, nurturing, and caregiving. ${ }^{2}$ For First Nations, taking care of one's physical body as the 'home of one's spirit' is important for physical wellness. ${ }^{2}$ Métis women linked physical wellness to healthy diet, being physically active, and being within a safe, clean environment. ${ }^{8}$

Mental wellness is described by the FNMWC as intuitive and rational thought and the understandings that are derived as a result of a balance between the two. ${ }^{2}$ Métis women identified intellectual and mental well-being as learning new things, being curious, and keeping an active mind. ${ }^{8}$ This intellectual and cognitive approach considered mental wellness to be related to having a sense of mean- 
ing of life and thinking about one's place in the world, as well as having intuition or 'inner knowing'. ${ }^{2}$ Inuit have various terms for problems with a person's state of mind, including isumaaluttuq, meaning "having heavy thoughts", "being preoccupied", or "thinking too much". ${ }^{11}$

\section{CONNECTIONS TO COMMUNITY}

Well-being is not only an individual issue, but is typically connected to the community. This included the importance of having strong social networks within one's cultural or geographic community to maintain mental wellness. ${ }^{13}$ The feeling of belonging was identified as important for First Nations, as traditional community living typically included living with extended families and multiple generations. ${ }^{4,9}$ The FNMWC relates emotional well-being to the feeling of belonging and connection to families and communities. ${ }^{2}$ Mussell refers to the importance of "wholeness, balance, the importance of relationships with family, community, ancestors, and the natural environment". ${ }^{10}$ Thus, well-being is not merely an individual concern, but a collective process whereby community members share in the responsibility of resolution and continual support during crisis. ${ }^{10}$ This is a marked difference to the Western concept of mental health, which focuses on dysfunction and individual coping strategies. ${ }^{10}$

A significant part of this collective process is dependent on intergenerational learning - having Elders and other adults as role models and teachers for children and young adults. ${ }^{9}$ This was thought to occur naturally from traditional extended family and clan system ways of living. ${ }^{2,9}$ Stewart describes the importance of being able to rely on others, including family, relatives, co-workers and even other communities for promoting good mental health. ${ }^{13}$ Likewise, Inuit people mentioned the importance of family and kinship, including sharing time together, carrying out daily activities together, talking about problems, visiting Elders regularly, hugging, and ensuring family members were happy. ${ }^{14}$

\section{LAND, CULTURE, AND WELLNESS}

Lack of well-being was seen as a result of disconnection from culture. ${ }^{15}$ Remedies for this lack of balance included returning to the land and taking part in the traditional way of life. ${ }^{16}$ Inuit Elders identified "going on the land, hunting, camping, eating country food, spending time with elders, making traditional tools, skin clothing, building an igloo, and Inuit beliefs and cosmology" as contributors to wellness. ${ }^{14}$

Kant and colleagues identified the importance of land use for Indigenous well-being, along with traditional diets, community self-governance, and access to cultural sites. ${ }^{17}$ The inherent connection of traditional food with community is further highlighted by the FNMWC, which describes the nurturing from Mother Earth that is gained from consuming and sharing traditional food in feasts, which reinforces bonds between individuals, families, and communities. $^{2}$

\section{WELLNESS AND INDIGENOUS IDENTITY}

Among First Nations, communal and ceremonial events also allowed for the sharing of important values and customs, which were seen as important aspects of wellness. ${ }^{2}$ For youth, learning from Elders about history, language, culture, and stewardship of the land was seen as helping youth gain a sense of pride in their identity, and combating feelings of shame and inferiority resulting from colonial domination. ${ }^{12,15}$ Having cultural identity as part of one's personal identity was thought to contribute to mental wellness. ${ }^{11}$ First Nations Elders described the importance of knowledge about culture and heritage and their use in building positive self-concept. ${ }^{12} \mathrm{Com}$ munity self-determination or self-governance were empowering for individuals to make decisions for their well-being with the help of their families, communities, Elders, and healers. ${ }^{7,9}$

\section{CONCLUSIONS FOR PRACTICE}

This brief review provides some additional understanding of the meanings of "holistic" as it relates to the wellness of Indigenous peoples. Many of the sources described the fundamental connections between aspects of individual wellness and the importance of community, traditional culture and activities, traditional lands and waters, and pride in an Aboriginal identity as contributors to Indigenous conceptions of mental wellbeing. We draw three main recommendations for physicians working with Indigenous populations.

On a cautionary note, providers should be aware of the diversity of Indigenous populations, including differences in cultural approaches to health. There is a great deal of cultural and linguistic diversity among Indigenous populations, as well as variation in personal and community histories and degrees of cultural connectedness. Although the literature revealed some themes that are potentially important for healthcare providers, these will not apply to everyone equally. Given this caution, we suggest that healthcare providers start by firstly being willing to ask new patients/clients how they identify themselves. We think that doing so would not only help guide the healthcare provider, but also acknowledge to patients that their Indigenous identity is considered an important aspect of their lives.

Second, where possible, healthcare providers should consider not only the immediate issues that may be affecting their Indigenous patients' mental health but also the historical, political, and social factors that can impact health. Recognizing the personal and intergenerational effects of colonization and the holistic nature of mental wellness is essential for healthcare providers. Furthermore, given the importance placed on intergenerational reliance and connectedness, whenever possible, opportunities to include families and Elders in healthcare discussions and treatment should be offered.

Finally, as outlined in the review, individuals can derive very real benefits from participating in traditional activities- spiritual prayer, ceremonies, or connecting with food and land of their ancestors. Considering this, we encourage healthcare providers with Indigenous patients/clients to make connections in their area of 
practice with Aboriginal Health Centres, or local Elders and healers who can provide culture-based counseling or healing for their patients. More importantly, we recommend healthcare providers offer these connections to patients as a component of their overall care.

\section{REFERENCES}

1. Government of Canada. The Mental Health and well-being of Aboriginal peoples in Canada. Ottawa, ON: Minister of Public Works and Government Services Canada, 2006.

2. Assembly of First Nations, Health Canada. First Nations Mental Wellness Continuum Framework. Ottawa, ON: Minister of Health; 2015. p. $1-64$.

3. Kirmayer LJ, Breass GM, Tait CL. The mental health of Aboriginal peoples: Transformations of identity and community. Can J Psychiatry. 2000;45(7):607-16. PubMed PMID: 11056823.

4. Mussell B, Cardiff K, White J. The Mental Health and Well-Being of Aboriginal Children and Youth: Guidance for New Approaches and Services. BC: Sal'i'shan Institute and The University of British Columbia, 2004.

5. National Aboriginal Health Organization. An Annotated Bibliography: Cultural Intervention Models in Mental Health. 2006.

6. Kirmayer LJ, Valaskakis GG, editors. Healing traditions: The mental health of Aboriginal peoples in Canada. Vancouver, B.C.: UBC Press 2009.

7. Mental Health Evaluation and Community Consultation Unit, Aboriginal Mental Health Committee, Adult Mental Health Division, Ministry of Health Services, Syme V, Mussell B. Aboriginal mental health- what works best? A discussion paper. B.C.: 200.

8. Bartlett JG. Health and well-being for Métis women in Manitoba. Can J of Public Health 2005;96 (Supplement 1):S22-S7. PubMed PMID: 15686149

9. Blackstock C. Rooting Mental Health in an Aboriginal World View: Inspired by Many Hands One Dream. 2008.

10. Mussell B. Restoration of Well-being for Canada's First Peoples. n.d.

11. Kirmayer LJ, Fletcher C, Watt R. Locating the ecocentric self: Inuit concepts of mental health and illness. In: Kirmayer LJ, Valaskakis GG, editors. Healing traditions: The mental health of Aboriginal peoples in Canada Vancouver, B.C. : UBC Press 2009. p. 289-314.

12. Nelson SE. Don't call me crazy: Re-envisioning mental health services for Aboriginal peoples in Prince George. Library and Archives Canada: University of Northern British Columbia; 2012.

13. Stewart SL. Promoting Indigenous mental health: Cultural perspectives on healing from Native counsellors in Canada. Int J Health Promot Educ. 2008;46(2):49-56. doi: 10.1080/14635240.2008.10708129.

14. Kral MJ, Idlout L, Minore JB, et al. Unikkaartuit: Meanings of well-being, unhappiness, health, and community change among Inuit in Nunavut, Canada. American Journal of Community Psychology. 2011;48(34):426-38. doi: 10.1007/s10464-011-9431-4. PubMed PMID: 21387118.

15. Vukic A, Gregory D, Martin-Misener R, et al. Aboriginal and Western conceptions of mental health and illness. Pimatisiwin: A Journal of Aboriginal and Indigenous Community Health. 2011;9(1):65-86.

16. Kirmayer L, Simpson C, Cargo M. Healing traditions: Culture, community and mental health promotion with Canadian Aboriginal peoples. Austral Psychiatry. 2003;11:S15-S23.

17. Kant S, Vertinsky I, Zheng B, et al. Social, cultural, and land use determinants of the health and well-being of Aboriginal peoples of Canada: A path analysis. J Public Health Pol. 2013;34(3):462-76. doi: 10.1057/ jphp.2013.27. PubMed PMID: WOS:000322378100009. 